\title{
Constant Fidelity Entanglement Flow in Quantum Communication Networks
}

\author{
Tan Bacinoglu Burhan Gulbahar Ozgur B. Akan \\ Next-generation Wireless Communications Laboratory \\ Department of Electrical and Electronics Engineering \\ Middle East Technical University, 06531, Ankara, Turkey \\ Email:\{tbacinoglu, bgulbahar, akan\}@eee.metu.edu.tr
}

\begin{abstract}
Entanglement distribution over long distances is one of the main problems in the existing quantum communication networks. Most of the existing methods of establishing entanglement paired link (Einstein, Podolsky, Rosen - EPR pairs) between distant nodes assume symmetric network topologies comprised of links with identical EPR generation capacities. In this work, the entanglement rate capacity of randomly distributed quantum ad hoc networks is investigated. To this end, constant fidelity maximum flow (CFMF) of entanglement problem is defined, and its theoretical analysis is presented. A new heuristic algorithm, i.e., Entanglement Swapping Scheme Search (ESSS), is presented to find the best possible swapping scheme over a multi-hop entanglement path. Furthermore, Shortest Path Entanglement Flow (SPEF) algorithm is introduced as an effective heuristic solution for this problem. Analysis shows that there is a trade-off between the desired constant target fidelity and the entanglement generation rate (maximum flow) of the network.
\end{abstract}

Index Terms-Quantum communication networks, EPR pairs, entanglement flow, swapping, purification, constant fidelity.

\section{INTRODUCTION}

Q UANTUM communication deals with the problem of transferring quantum states from one location to another. The most common method to transfer quantum states, is quantum teleportation [1], which exploits entanglement phenomena to establish quantum channels by EPR pairs [2], [3]. At present, entanglement generation (EG) via fibers or optical free space links is possible for distances on the order of hundred kilometers, e.g., $250 \mathrm{~km}$ on fiber links [4] and $144 \mathrm{~km}$ on free space [5]. However, due to photon loss and detector noise, the success rate of the entangled photon generation decreases exponentially with distance [6]. This makes the long-distance EG an important challenge for quantum communication.

One solution is to use quantum repeaters $(\mathrm{QR})$ between source and destination nodes [7]. QRs are quantum communication nodes capable of storing quantum states and performing basic quantum operations coordinated by QR protocols. In general, a QR protocol involves the sequential application of link-level entanglement generation, entanglement swapping (ES) and entanglement purification (EP) [7], [8] operations. The main goal of QR protocols is to achieve the highest possible success rate for link establishment while keeping the

This work was supported in part by the Turkish Scientific and Technical Research Council Career Award under grant \#109E257, by the Turkish National Academy of Sciences Distinguished Young Scientist Award Program (TUBA-GEBIP), and by IBM Faculty Award. fidelity of pairs within acceptable levels and making longdistance entanglement efficiently realizable.

Even with QR technology, long-distance EG is still a challenging problem due to the inevitable presence of imperfections, e.g., memory decay, local measurement errors and ES or EP failures [9], [10]. These imperfections and the finite link-level EPR generation capacity determine an upper bound for the EG rate between nodes connected by QRs. Clearly, they determine a two-way quantum communication capacity that limits maximum achievable throughput in both directions and QR protocols should be optimized to reach this limit.

In the literature, there are some works on the optimization of QR protocols [11], [12], [13]. In addition, some classical network concepts are adapted to quantum network domain as well [6], [15], [8], [14]. In these studies, only symmetric network topologies are investigated, which is an unrealistic assumption for future quantum networks. Nevertheless, an entanglement generation capacity analysis for EPR distribution over randomly distributed quantum ad hoc networks has not been considered yet.

In this paper, an EPR generation rate capacity analysis over randomly distributed quantum ad hoc networks is performed and constant fidelity maximum flow (CFMF) of entanglement problem is defined. The average EPR generation rate on a multi-hop path is derived depending on the specific ES scheme in use. A new heuristic algorithm is introduced, i.e., Entanglement Swapping Scheme Search (ESSS), which aims to find the best possible ES scheme over a multi-hop path between a single source and sink in a quantum ad hoc network. Furthermore, a new algorithm, i.e., Shortest Path Entanglement Flow (SPEF), is proposed to obtain an effective solution for CFMF problem. Simulation studies show that there is a trade-off between the entanglement flow rate and the desired constant target fidelity.

The remainder of the paper is organized as follows. In Section II, a brief review of related work is presented. In Section III, the EPR generation capacity analysis for a single and multi-hop links established by different ES schemes are derived, and the details of ESSS algorithm are introduced. In Section IV, CFMF problem is defined and the operational details of SPEF algorithm are presented. Numerical analysis results in Section V are followed by concluding remarks discussed in Section VI. 


\section{RELATED WORK}

The most relevant existing related work on the networking aspects of long-distance quantum communication networks (QCNs) can be reviewed in two categories as follows.

\section{A. Optimization of $Q R$ Protocols}

QR protocols are optimized to obtain faster EPR generation rates or improved final fidelity of the EPR pairs. In [11], QR protocol optimization is achieved by using dynamical programming and multi-level pumping mechanism is introduced nesting several levels of entanglement pumping in order to increase the fidelity of generated pairs.

In a QR stack, a banded EP algorithm is presented for low fidelity EPR pairs supporting the experimental aspects of wide area QCNs and the system performance is increased with better utilization of physical and temporal resources [12].

A QR protocol is presented in [13], where memory coherence time does not limit the communication distance by encoding qubits with Calderbank-Shor-Steane (CSS) codes and using classical error correction instead of time consuming quantum error correction and EP operations.

However, these works consider only the case where QR links have identical capacities. In fact, QRs may be adjusted to connect not only two predetermined end nodes but the whole network. Hence, link capacities are expected to be nonidentical in future QCNs as in classical networks.

\section{B. Classical Networking in Quantum Communications}

A flexible qubus repeater protocol architecture is presented in [6]. It provides quantum communication capability using coherent states with flexible memory and local input states and enables high quality and faster long distance EG.

Novel quantum broadcasting schemes like ringular, angular, and regional broadcast unique to the QCNs are defined in a QR architecture [8] extending the classical broadcasting concepts.

A novel entanglement generation and connection architecture is presented in [14] using multiplexed memory elements by improving communication rates for quantum networks with short memory coherence times.

Unlike the existing related studies reviewed above, in this work, entanglement flow generation capacity problem is investigated for randomly distributed quantum ad hoc network topologies with links having nonidentical capacities.

\section{EPR GENERATION CAPACITY OVER QUANTUM PATHS}

Here, we first present a capacity description for link-level EPR generation, and then, extend these definitions to obtain an EPR generation rate formulation for multi-hop paths.

\section{A. Capacity of EPR generation and Entanglement Connection}

The average time, $\tau_{i j}$, in which an EPR pair can be generated between two consecutive repeater nodes $i$ and $j$, i.e., an elementary link, is expressed as the sum of the detection, i.e., $1 / f P_{g}$, and communication time [6], i.e., $d_{i j} / c$,

$$
\tau_{i j}\left(f, d, P_{g}\right)=1 / f P_{g}+d_{i j} / c
$$

where $f$ is the operation frequency, $P_{g}$ is the success probability of realizing an EPR pair and $c$ is the speed of light. $P_{g}$ depends on the distance $d_{i j}$ between repeaters $i$ and $j$, and the fidelity of the entangled pair $F$ between nodes, which is expressed by [6]

$$
P_{g}(F)=0.5\left[1-(2 F-1)^{\frac{2 \eta}{1-\eta}}\right]
$$

where $\eta=e^{-\xi d}$ is the loss parameter over the distance $d$ with the loss rate $\xi$. Moreover, the operation frequency $f$ is limited by the quantum memory space [6]

$$
M=4\lceil d f / c\rceil
$$

As seen in (2) and (3), EPR generation time on an elementary link depends on both link distance $d$ and memory space $M$. Memory space determines the maximum operation frequency $f_{\max }=M c / 4 d$ and link distance determines communication time $\frac{d}{c}$ and the success probability $P_{g}(F)$.

Typically, the detection time is much longer than the communication time, i.e., $\frac{1}{f P_{g}} \gg \frac{d}{c}$, and hence, the average EPR generation time is approximately equal to the detection time $\frac{1}{f P_{g}}$. Therefore, EPR generation capacity of an elementary link can be defined by its maximum detection rate, i.e.,

$$
C(F) \equiv f_{\max } P_{g}(F)
$$

Accordingly, an elementary link can generate EPRs with an average rate below this capacity by operating at a frequency below the maximum operation frequency $f_{\max }$.

In order to be able to estimate the average EPR generation rates on joint QR links, we define the entanglement connection (EC) operation as the successive operations of ES and EP that preserve the constant fidelity level of EPR pairs. This is possible if EP and ES operations are used in an intrinsically balanced manner to satisfy a constant fidelity condition over all the formed EPR links. This condition is required to make the EC operation more consistent with the self-similar nature of the QR structures. Hence, EPR links formed by EC can be switched to be used on different $\mathrm{QR}$ paths.

Suppose two elementary links $(A, B)$ and $(B, C)$ are to be combined by EC to maintain the constant fidelity $F$ as shown in Fig. 1. Let $R(i, j)$ be the EPR generation rate achieved between the QRs $i$ and $j$. The first link generates EPR pairs with an average rate of $R(A, B)=f_{1} P_{g, 1}(F)$ and the second generates with an average rate of $R(B, C)=f_{2} P_{g, 2}(F)$. Here, we assume that both links start operating at the same time and wait for an EC operation whenever an EPR pair is ready. Thus, the average time required to obtain an EPR pair on both links is lower bounded by $\max \left\{\frac{1}{f_{1} P_{g, 1}(F)}, \frac{1}{f_{2} P_{g, 2}(F)}\right\}$. Since EC operation also has its own probability of success $P_{c}$ [6], this procedure should be repeated $1 / P_{c}$ times, on the average, in order to achieve successive connection. Therefore, the minimum average time $\tau_{A, C}$ to obtain an EPR pair between $A$ and $C$ can be expressed as

$$
\tau_{A, C}=\max \left\{\frac{1}{f_{1} P_{g, 1}(F)}, \frac{1}{f_{2} P_{g, 2}(F)}\right\} \frac{1}{P_{c}}
$$

In the same manner, the maximum average EPR pair generation rate $R(A, C)$ can be expressed as

$$
R(A, C)=\min \{R(A, B), R(B, C)\} P_{c}
$$




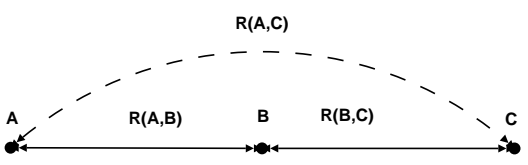

Fig. 1. EPR generation by combining two links with different rates.

Next, the rate analysis described here is generalized for multihop entanglement flow paths.

\section{B. EPR Generation Rate on Multi-hop Entanglement Paths}

Let the last EC operation be realized on the $k^{\text {th }}$ repeater on an $N$-hop quantum repeater path, where the total number of nodes including source and destination is $N+1$. Then, the EPR generation rate achieved on this path can be given as

$$
R(0, N)=\min \{R(0, k), R(k, N)\} P_{c}
$$

Alternatively, $R(0, N)$ can be written by recursively replacing $R(i, j)$ terms with their explicit expressions as in the form.

$$
R(0, N)=\min \left\{R(i, i+1) P_{c}^{n(i, i+1)}\right\}
$$

where $n(i, i+1)$ is the nesting level of the link $(i, i+1)$, i.e., the total number of ES operations performed on the link.

Consider the 3-hop network given in Fig. 2(a). There are two ES schemes that can be used to establish an EPR link between the nodes 0 and 3 . The resulting EPR generation rate depends on which swapping scheme is used, it could be one of the following two schemes, i.e., for Scheme-1

$$
R(0,3)=\min \left\{R(0,1) P_{c}, R(1,2) P_{c}^{2}, R(2,3) P_{c}^{2}\right\}
$$

and, for Scheme-2,

$$
R(0,3)=\min \left\{R(0,1) P_{c}^{2}, R(1,2) P_{c}^{2}, R(2,3) P_{c}\right\}(10)
$$

If the lowest rate is $R(1,2)$, both schemes give the same EPR generation rate, i.e., $R(0,3)=R(1,2) P_{c}^{2}$. However, if the lowest rate is one of $R(0,1)$ or $R(2,3)$, they give different average EPR generation rates. For example, Scheme1 as in (9) is a better choice if the lowest rate is $R(0,1)$, since $\min \left\{R(0,1) P_{c}, R(1,2) P_{c}^{2}, R(2,3) P_{c}^{2}\right\}$ is higher than $R(0,1) P_{c}^{2}$. As a result, it can be concluded that connecting links with higher rates first is a better approach for an efficient ES strategy, because connecting links with lower rates should be avoided in each nesting level in order not to further reduce their rates by ES.

An ES scheme over a QR path can be also represented by a binary tree where leaf nodes correspond to elementary links which are the sources of EPR generation flow through the root node. For example, two ES schemes for a 3-hop path can be illustrated as in Fig. 2(b).

Furthermore, the total number of possible ES schemes over an $N$-hop repeater path can be described analytically as the following. Let $\varsigma_{N}$ be the set of all possible ES schemes for an $N$-hop quantum path. $\left|\varsigma_{N}\right|$, which is the cardinality of the set $\varsigma_{N}$, can be expressed in terms of $\left|\varsigma_{M}\right|$ where $M<N$ as

$$
\left|\varsigma_{N}\right|=\sum_{j=1}^{N-1}\left|\varsigma_{j}\right|\left|\varsigma_{N-j}\right|, \text { for } N>1
$$

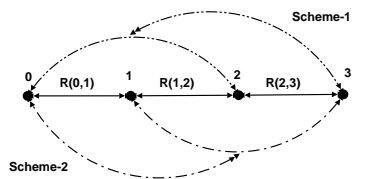

(a)

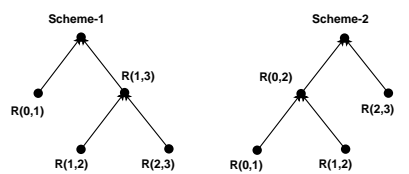

(b)
Fig. 2. (a) Two different ES schemes for a 3-hop QR path. (b) Binary tree representations of two ES schemes for a 3-hop QR path.

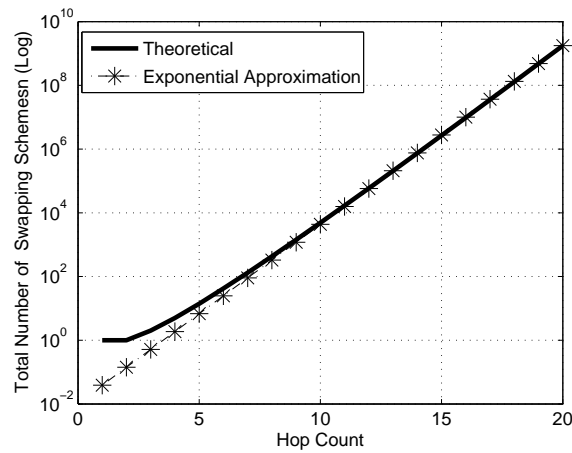

Fig. 3. Number of swapping Schemes vs. hop count.

Here $\left|\varsigma_{1}\right|=1$ and $\left|\varsigma_{N}\right|$ increases exponentially with hop distance $N$. In fact, for reasonable $N$ values until 20 hop counts $\left|\varsigma_{N}\right| \propto 0.0107 \times 3.6383^{N}$. This exponential dependence on hop distance and its approximation are shown in Fig. 3.

Moreover, the problem of finding the optimum swapping scheme can be generalized for multihop QR paths as follows. Let $\mathbf{C}_{\mathbf{P}}$ be the $N \times 1$ capacity vector of an $N$-hop quantum path $P$, where each entry $c_{i}$ is the EPR generaton capacity of the link $(i-1, i)$, and let $\mathbf{P}_{\mathbf{c}}$ be a $N \times N$ diagonal matrix where diagonal entries correspond to swapping coefficient $P^{n_{i}}$ describing the swapping scheme used to connect endnodes 0 and $N$. The maximum EPR generation rate that can be achieved using the swapping scheme defined by $\mathbf{P}_{\mathbf{c}}$ is the smallest element $R(0, N)$ of the after swapping capacity vector, i.e., $\mathbf{C}_{\mathbf{P}}^{\mathbf{s}}$, which can be written as

$$
R(0, N)=\min \left\{r_{i} \mid r_{i} \in \mathbf{C}_{\mathbf{P}}^{\mathbf{s}}\right\}
$$

where,

$$
\mathbf{C}_{\mathbf{P}}^{\mathrm{s}}=\mathbf{P}_{\mathbf{c}} \mathbf{C}_{\mathbf{P}}
$$

Then, the optimum swapping scheme can be defined as the swapping scheme described by $\mathbf{P}_{\mathbf{c}}$ that gives the maximum $R(0, N)$ value for a given capacity vector $\mathbf{C}_{\mathbf{P}}$. The optimum swapping scheme can be searched with a recursive algorithm, i.e., entanglement swapping scheme search (ESSS) algorithm.

The operation of ESSS algorithm given in Algorithm 1 is explained as follows. Given an $N$-hop quantum path $P$ and its capacity vector $\mathbf{C}_{\mathbf{P}}$, ESSS algorithm finds the maximum $R(s, t)$ value, i.e., $R_{\max }(s, t)$, between end-nodes $s$ and $t$. Basically, ESSS stores the label of the repeater node $j_{s t}$, connecting links $\left(s, j_{s t}\right)$ and $\left(j_{s t}, t\right)$, then modifies $j_{s t}$ according to the comparison of $R_{\max }\left(s, j_{s t}\right)$ and $R_{\max }\left(j_{s t}, t\right)$ values in each nesting level. The algorithm stops when the new value 


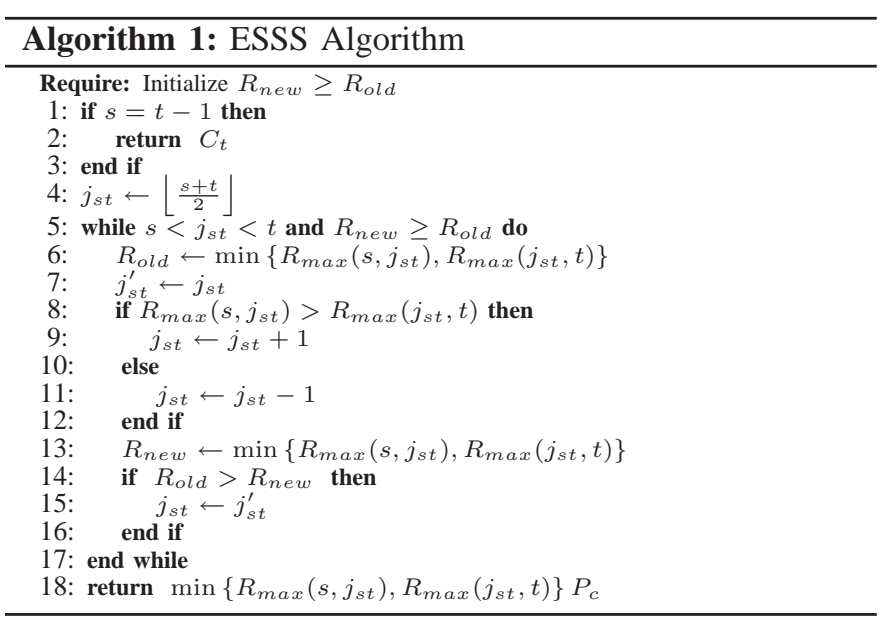

$R_{\text {new }}$ becomes smaller than the old value, $R_{\text {old }}$, meaning that the optimum swapping scheme is reached.

\section{Constant Fidelity Entanglement Flow}

Here, first, maximum entanglement connection problem is defined as an optimization problem, then, a SPEF algorithm is introduced as a heuristic solution to this problem.

\section{A. CFMF Problem Definition}

Constant fidelity maximum flow (CFMF) of entanglement problem can be described as follows. Given a connected graph $\mathrm{G}=(\mathrm{V}, \mathrm{E})$, where $V$ is a set of nodes, i.e., $Q R s$, and $E$ is a set of edges, i.e., elementary quantum links, between these nodes, the quantum capacity on the links and a pair $(s, t)$ of sourcesink nodes, find the maximum EPR generation rate between source $s$ and sink $t$.

Furthermore, it is possible to state the problem mathematically as the following. Let $\mathbf{W}$ be an $N \times N$ weighted adjacency matrix of an undirected graph where each entry $c_{i j}$ is the EPR generation capacity of the link. Let $P_{l}^{(s, t)}$ represent a specific path between the source node $s$ and destination $t$. The consumed capacity on the path $P_{l}^{(s, t)}$ in link $(i, j)$ is given by $c_{i j} \times u_{i j}$. Here, $u_{i j}$ are the elements of $\mathbf{U}_{\mathbf{P}^{(\mathbf{s}, \mathbf{t})}}$, which is the path capacity usage ratio matrix with size $N \times N$. Note that $u_{i j}$ values correspond to the non-zero entries of $\mathbf{W}$ and the sum of path capacity usage matrices $\mathbf{U}_{\mathbf{P}_{1}^{(\mathbf{s}, \mathbf{t})}}$ is an $s-t$ cut matrix on $\mathbf{W}$ where entries which equal to one correspond to an $s-t$ cut on $\mathbf{W}$ and the other entries are smaller than one. Let $\mathbf{C}_{\mathbf{P}_{1}^{(s, t)}}$ denote the swapping matrix with size $N \times N$ for the path $P_{l}^{(s, t)}$, where each non-zero entry $b_{i j}$ of the vector is the swapping coefficient $P_{c}^{n(i, j)}$ for the corresponding link on the path. Then, CFMF entanglement connection problem can be defined as

$\max \sum_{l} \min \left\{r_{k} \mid r_{k} \neq 0\right.$ and $\left.r_{k} \in \mathbf{W} \odot \mathbf{U}_{\mathbf{P}_{1}^{(\mathbf{s}, \mathbf{t})}} \odot \mathbf{C}_{\mathbf{P}_{1}^{(\mathbf{s}, \mathbf{t})}}\right\}$

s.t. $\quad \sum_{l} \mathbf{U}_{\mathbf{P}_{1}^{(\mathbf{s}, \mathbf{t})}}$ is an $s-t$ cut matrix on $W$.

Some heuristic approaches can be developed for this optimization problem based on algorithmic solutions such as

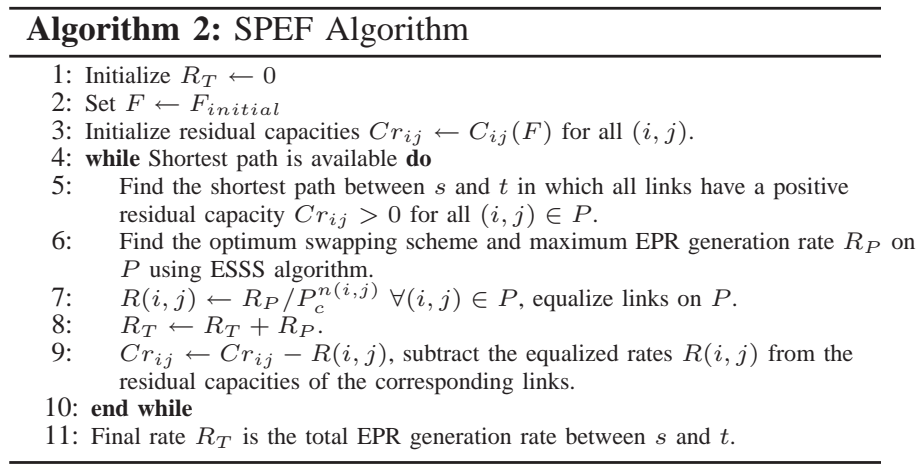

path searching and routing algorithms over the network. The complexity analysis of the problem and optimal solutions are left for future work. Next, a heuristic solution, i.e., SPEF algorithm is formulated, and its basics are presented.

\section{B. Shortest Path Entanglement Flow Algorithm}

CFMF problem is a modification of maximum flow problem for entanglement distribution over quantum networks.One crucial difference is that capacity usage on elementary links depends on the swapping schemes used on QR paths. For example, shorter QR paths use link capacities more efficiently than longer QR paths since each swapping decreases the average EPR link generation rate. Because of this, we propose to start filling link capacities by consuming them on shorter paths first. The SPEF algorithm that represents this approach is outlined in Algorithm 2.

One of the problems in the application of ES operations on multi-hop paths is the nonidentical values of capacities which results in wasting of higher capacity links because of the diminishing effect of the lower capacity links on the overall EPR generation rate. A practical solution is to try to balance the capacity values of the links over the multi-hop path, i.e., quantum path equalization. Equalization on a quantum path is the method of equalizing the reflected link rates $R(i, j) P_{c}^{n(i, j)}$ so that all nested ES operations on this path connect links with equal rates on average. Once this condition for equalization is satisfied, there will be no excessive use of quantum capacity on this path and the resulting rate will be equal to a reflected link rate. Hence, equalization is needed to achieve maximum EPR generation rate with minimum capacity and is used in SPEF algorithm.

\section{NumericAl ANALYSis}

We performed simulations to observe the achievable EPR generation rate (EPR pairs/second) between two nodes $(s, t)$ separated by $100 \sqrt{2} \mathrm{~km}$, and located on bottom-left and topright corners of a square area of $10000 \mathrm{~km}^{2}$, respectively. Operation frequencies of elementary links are $100 \mathrm{~Hz}$.

\section{A. EPR Generation Rate over Multi-hop Paths}

Here, in Fig. 4, we first show the maximum EPR generation rate variation with number of links for varying connection probability $P_{c}$ with fidelity $F$ set to different target constant 


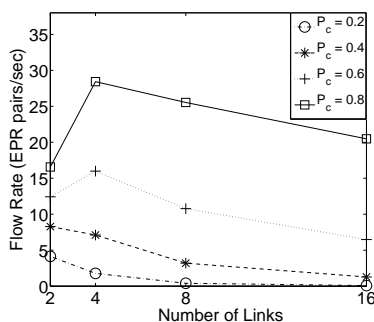

(a)

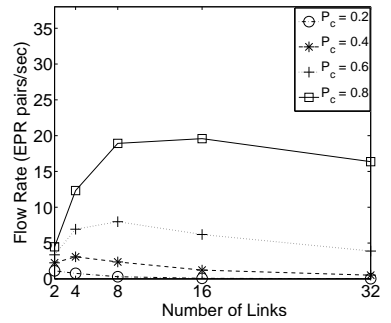

(c)

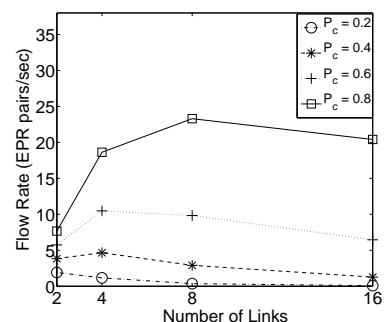

(b)

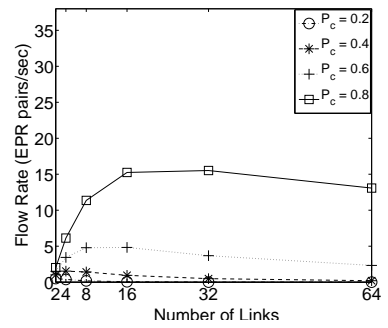

(d)
Fig. 4. EPR generation rate vs. number of links for varying $P_{c}$ when target constant $F$ is set to (a) $F=0.55$ (b) $F=0.7$ (c) $F=0.8$ (d) $F=0.9$.

values when all repeaters are aligned on the diagonal between nodes $s$ and $t$. It appears that as $P_{c}$ decreases, the number of links giving higher EPR generation rates becomes lower. In addition, as target fidelity level increases, the number of links giving higher EPR rate becomes higher for all $P_{c}$ values. The reason for this is due to the fact that with lower link capacities more repeaters are required to overcome the exponential decrease in EPR generation rate. However, as $P_{c}$ increases to 1 , the point for optimum number of links, i.e., peak point, moves to infinity. This is expected because in the limiting case, i.e., $P_{c}=1$, EPR generation rate becomes equal to EPR generation capacities of elementary links.

\section{B. EPR Generation Rate for Random Topology}

In the second group of experiments, repeater nodes between $s$ and $t$ are uniformly distributed on the square area. The flow rate between $s$ and $t$ is found using SPEF algorithm for each random realization of the spatial node distribution and the average flow rate value of 20 different random realizations are taken for each setup. Furthermore, a threshold value, i.e., connection threshold radius, is set for each random realization to avoid elementary links longer than this radius. With these configurations, we observed flow rate for varying target fidelity and connection radius with different node density values.

In Fig. 5(a), it is observed that the entanglement flow rate depends almost linearly on target constant fidelity. This may be due to the fact that effective elementary node distance is on the linear portion of the fidelity versus $P_{g}$ curve. In Fig. 5 (b), it can be easily seen that connection threshold radius significantly affects the flow rate. This observation reflects the fact that increasing connection threshold radius makes shorter paths available and increases the connectivity of the quantum network by adding more capacity. However, the flow rate saturates after some value of connection threshold radius which corresponds to the maximum connectivity distance (a)

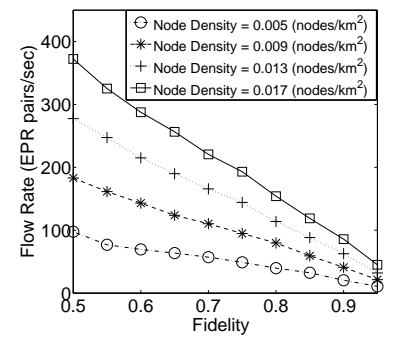

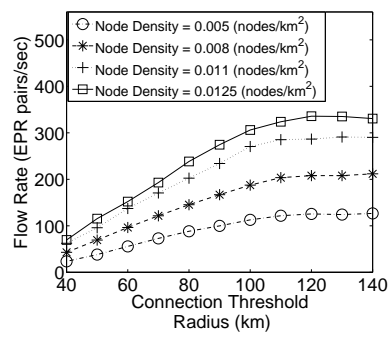

(b)
Fig. 5. Flow rate vs. (a) target $F$, connection thresh. radius $=50 \mathrm{~km}$, (b) connection thresh. radius, target $F=0.8$; for varying node density, $P_{c}=0.5$

among any two nodes in our setup, i.e., $100 \sqrt{2} \mathrm{~km}$. In both cases, increasing node density directly increases the total capacity, however, the effect of adding more nodes decreases with either increasing fidelity or decreasing connection radius due to their decreasing effect for the network connectivity.

\section{CONCLUSION}

In this work, the entanglement rate capacity of randomly distributed quantum ad hoc networks is investigated. Constant fidelity maximum entanglement flow generation problem is defined, and its theoretical analysis is presented. Furthermore, two new heuristic algorithms, i.e., ESSS and SPEF, are introduced for this problem. Numerical analysis results show that the target constant fidelity level dominantly affects the achievable entanglement generation rate of the network.

\section{REFERENCES}

[1] C. Bennett, et. al., "Teleporting an unknown quantum state via dual classical and EPR channels", Phys. Rev. Lett., vol. 70, no. 13, 1993.

[2] A. Einstein, B. Podolsky, N. Rosen, et. al., "Can quantum-mechanical description of physical reality be considered complete?", Phys. Rev., vol. 47, no. 10, pp. 777-780, 1935.

[3] R. Horodecki, P. Horodecki, M. Horodecki, and K. Horodecki, "Quantum entanglement”, Rev. Mod. Phys., vol. 81, no. 2, pp. 865-942, 2009.

[4] D. Stucki, et. al., "High rate, long-distance quantum key distribution over $250 \mathrm{~km}$ of ultra low loss fibres", New Journ. Phys., vol. 11, 2009.

[5] T. Schmitt-Manderbach, et. al., "Experimental demonstration of freespace decoy-state quantum key distribution over 144 km", Phys. Rev. Lett., vol. 98, no. 1, p. 10504, 2007.

[6] B. He, Y.-H. Ren, J. A. Bergou, "Creation of high-quality long-distance entanglement with flexible resources", Phys. Rev. A, vol. 79, 2009.

[7] W. Dür, H. Briegel, J. Cirac, P. Zoller, "Quantum repeaters based on entanglement purification”, Phys. Rev. A, vol. 59, no. 1, 1999.

[8] B. Gulbahar and O. B. Akan, "Broadcast routing in quantum ad hoc networks", in Proc. IEEE Nanocom 2009, USA, Aug. 2009.

[9] H.-J. Briegel, et. al., "Quantum repeaters: The role of imperfect local operations in quantum communication", Phys. Rev. Lett., vol. 81, 1998.

[10] L. Hartmann, B. Kraus, H.-J. Briegel, and W. Dür, "Role of memory errors in quantum repeaters", Phys. Rev. A (Atomic, Molecular, and Optical Physics), vol. 75, no. 3, p. 032310, 2007.

[11] L. Jiang, J. Taylor, N. Khaneja, and M. Lukin, "Optimal approach to quantum communication using dynamic programming", Proceedings of the National Academy of Sciences, vol. 104, no. 44, p. 17291, 2007.

[12] R. V. Meter, T. D. Ladd, W. J. Munro, and K. Nemoto, "System design for a long-line quantum repeater", ArXiv e-prints, May 2007.

[13] L. Jiang, et. al., "Quantum repeater with encoding", Phys. Rev. A, vol. 79, no. 3, p. 032325, 2009.

[14] O. A. Collins, et. al., "Multiplexed memory-insensitive quantum repeaters", Phys. Rev. Lett., vol. 98, p. 060502, 2007.

[15] L. Duan, et. al., "Long-distance quantum communication with atomic ensembles and linear optics", Nature, vol. 414, 2001. 